vol.1 No.1 ISSN:18235-2014

\title{
Early Initiation of Gn RH Antagonist versus long agonist Protocols in PCOS Patients Undergoing ICSI Cycles.
}

\author{
By \\ ${ }^{1}$ Sameh El-Azab , ${ }^{2}$ Mohammad Emam, ${ }^{3}$ Hamed Youssef, \\ ${ }^{4}$ Abu-El-Hasan El-Shazly and ${ }^{5}$ Ekbal Abou-Hashem \\ 1,2,3,4 Department of Obstetrics and Gynecology, Faculty of Medicine ,Mansoura University \\ 5 Department of Clinical Pathology, Faculty of Medicine, Mansoura University, \\ E. mail of the corresponding author:dr samah-elazab@yahoo.com
}

\begin{abstract}
Purpose: To compare the efficacy of early initiation of Gonadotrophin Releasing Hormone (GnRH) antagonist in comparison with the GnRH agonist protocol in Polycystic Ovarian Syndrome (PCOS) patients undergoing Intracytoplasmic Sperm Injection (ICSI) cycles. Materials and methods: Prospective randomized controlled trial. Seventy infertile PCOS patients under 35 years of age coming for the first trial IVF/ICSI treatment at the infertility care unit were included in the study. They were randomized to either GnRH agonist long protocol (control group A) or early initiation of GnRh antagonist (study group B) after pretreatment with OCP. Ovulation was triggered with Humman Chorionic Gonadotrophin (hCG) when at least 3 mature follicles $18 \mathrm{~mm}$ were detected. Results: Age, body mass index, duration of infertility, basal Follicle Stimulating Hormone (FSH), Lutinizing Hormone (LH), Estradiol (E2) at the first day of the cycle, the number of oocytes retrived, the total number of embryos, chemical, clinical pregnancy and implantation rates were comparable in both groups and there was no statistically significant difference. The stimulation period was shorter in group B than group A and number of ampoules of gonadotrophins ampoules and Serum E2 level on the day of hCG administration were significantly lower in group B than that of the group A with statistically significant differences. Moderate OHSS was documented in 4 cycles of group A but was not in any cycle of group B and this difference was statistically significant. No cases of severe OHSS were documented in both groups.Conclusions: This novel antagonist protocol may be a safe and efficient treatment for PCOS patients undergoing ICSI cycles with comparable results to the standard long GnRH agonist protocol.
\end{abstract}

key wards PCOS, ICSI, Gonadotrophin Releasing Hormone (GnRH) antagonist

Introduction:

Polycystic ovary syndrome (PCOS) is the most common cause of anovulatory infertility, affecting approximately 5\%$10 \%$ of reproductive-age women. Approximately $85 \%$ of these women have elevated levels of Luetinizing hormone (LH) resulting in the arrest of ovarian follicular growth ${ }^{(1)}$. The optimal infertility treatment for PCOS women is still a matter of controversy. In 2008, a consensus was reached on treatment for PCOS patients that includes the use of clomiphene citrate, exogenous gonadotrophins, laparoscopic ovarian surgery and in vitro fertilization (IVF) ${ }^{(2)}$. A proportion of PCOS women do not respond to the conventional treatments and ultimately require assisted reproductive technology (ART). Ovarian stimulation in women with PCOS poses a particular challenge, as many of these women exhibit exaggerated response ${ }^{(3)}$.

Since the early 1980s, the GnRH agonists in ovarian stimulation were used to reduce the incidence of premature LH surges by suppressing gonadotrophin release via pituitary desensitization following an initial short period of gonadotrophin hypersecretion. Since 1999, GnRH antagonists with high potency and fewer side effects have been introduced into IVF and have emerged as an alternative in preventing premature LH surges ${ }^{(4)}$.

$\mathrm{GnRH}$ agonists and GnRH antagonist were used in PCOS patients undergoing IVF/ICSI for controlled ovarian stimulations with variable results. But none of these until now is ideal for these patients. Most of the randomized control trials (RCT) comparing GnRH antagonists versus agonists have employed either a fixed (starting on day 6) or a flexible dose (starting when the leading follicle reaches $14-15 \mathrm{~mm})^{(5)}$.

One of the currently debatable issues regarding the use of $\mathrm{GnRH}$ antagonists refers to the timing of $\mathrm{GnRH}$ antagonist initiation. Research for alternative $\mathrm{GnRH}$ antagonist protocols regarding the timing of its initiation has 
shifted towards earlier antagonist initiation than Day 6 of stimulation ${ }^{(6)}$.

There is a theoretical benefit in the addition of GnRH antagonists to controlled ovarin hyperstimulation $(\mathrm{COH})$ protocols, especially in patients with PCOS with high LH levels, during the follicular phase. But profound inhibition of endogenous $\mathrm{LH}$ at early stage may result in faulty folliculogenesis ${ }^{(7)}$.We performed this prospective randomized controlled trial to compare the early initiation antagonist protocol with the standard GnRH agonist long protocol for PCOS patients undergoing ICSI treatment.

\section{Subjects and Methods:}

\section{Study design:}

The present study is a prospective randomized controlled study conducted at Mansoura University Hospital, Fertility Care Unit during the period from October 2009 to December 2012. The patients were randomly allocated into two groups by using computer-generated randomization: group A (agonist group) and group B (antagonist group). All Participants signed an informed consent after explaining the two protocols of controlled ovarian hyperstimulation to them.

\section{Patient population}

Seventy Patients with PCOS (based on the Rotterdam consensus criteria) who have PCO by Ultrsound, under 35 years of age coming for the first trial IVF/ICSI treatment at the infertility care unit were included in the study. Both groups (group $\mathrm{A}$ and $\mathrm{B}$ ) were matched as regard age, body mass index (BMI) and duration of infertility. Additional inclusion criteria are: basal serum FSH of $<10 \mathrm{mIU} / \mathrm{ml}$, uterine cavity without abnormalities and normal semenogram according to the WHO criteria (WHO, 1999). Patients with known previous poor ovarian response were excluded.

Ovarian stimulation and_Monitoring of the cycle

All patients received oral contraceptive pill (OCP) contained $30 \mu \mathrm{g}$ ethinyl E2 and 75 $\mu \mathrm{g}$ gestodene (Gynera, Bayer Schering,
Germany), starting on Day 2 of Spontaneous or induced menses of the cycle prior to the treatment cycle, after Blood test confirmed the presence of a baseline hormone profile.

Patients in GnRH agonist group (group A) received the standard long GnRH agonist protocol. Triptorelin (Decapeptyl@, Ferring, Germany) $100 \mu \mathrm{g}$ was given via subcutaneous injection starting 3 days before discontinuation of the oral contraceptive. On day 2 of menstrual cycle (day 1 of stimulation), a daily injection of HMG (Merional@, IBSA, swizerland)was administrated at 225 IU IM After documentation of pituitary desensitization (absence of ovarian follicles of $>4 \mathrm{~m}$ and the absence of a thick endometrium on transvaginal ultrasound examination and serum estradiol $<50 \quad \mathrm{pg} / \mathrm{ml}$ ) The administration of Triptorelin (Decapeptyl@, Ferring, Germany) was continued until the day of $\mathrm{HCG}$ administration.

In the GnRH antagonist group (group B), patients started daily HMG (Merional@, IBSA, swizerland) at 225 IU IM on Day 1 of stimulation. Subcutaneous cetrorelix (Cetrotide@, Serono) 0.125 mg (0.5 ampoule) was given daily concomitantly with HMG from the Day 1 of stimulation up to the leading follicle reaches $14 \mathrm{~mm}$. At this time subcutaneous cetrorelix (Cetrotide@, Serono) 0.25 mg (1ampoule) was given daily until the day of hCG administration.

In both groups, serial transvaginal ultrasound examinations using vaginal probe were performed to monitor the follicular growth. The starting dose of HMG was then adjusted individually depending on the ovarian response, as assessed by E2 levels and ultrasound. A step-down protocol was used, if necessary. Endometrial thickness was also assessed on the day of HCG administration.

Ovulation was triggered with 10000 IU of IM hCG (Choriomon@, IBSA, swizerland) when at least three mature follicles $18 \mathrm{~mm}$ were detected on ultrasound scan. 
Transvaginal ultrasound guided oocyte retrieval was done. All patients were scheduled for ICSI. Embryo transfer procedure was arranged 48-72 hours after oocyte retrieval. Grade A and B embryos were transferred under transabdominal ultrasound guidance. All patients received luteal phase support with I.M. progesterone 100 mg (Prontogest@, IBSA, AMSA, Italy) daily starting on the day of oocyte retrieval.

\section{Outcome measures}

The primary outcome measures were the number of gonadotrophins ampoules used per each cycle, the duration of stimulation, number and degree of maturity of oocytes retrieved, rate of fertilization and available embryo for transfer. While secondary outcome measures were implantation rate, incidence of OHSS

\section{Statistical analysis}

Statistical analysis was carried out via both Statistical Package for Social Science
(SPSS) version 17 on windows XP. Qualitative data were represented in the form of number and frequency, while quantitative data were represented in the form of mean \pm standard deviation (mean $\pm \mathrm{SD})$. Kolmogrov-smirnov test was used to test normality of quantitative data, and some data were non-normally distributed. Student's $t$ test, Mann-Whitney and chi square tests were used to compare groups.

The difference is considered significant if $P$ value is less than or equal 0.05 .

\section{Results:}

Seventy patients were initially randomized but three patients in group A and four patients in group B were lost follow up. There were four patients in group A and two patients in group B cancelled early due to exaggerated ovarian response and did not complete the follow up after counseling the couple regarding the risk of lifethreatinig OHSS

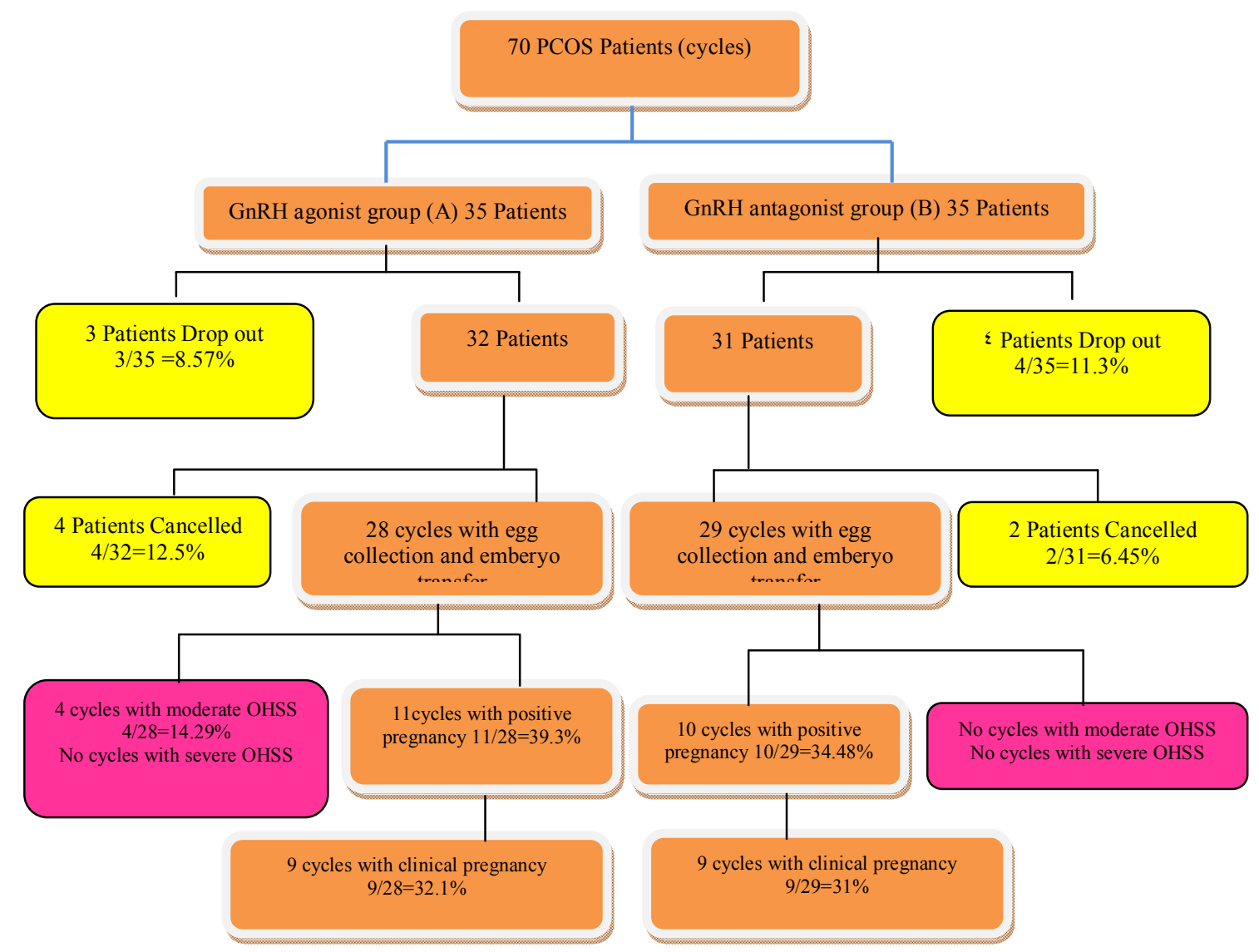

Flow chart for the study 
Sameh El-Azab et.al.

\begin{tabular}{|c|c|c|c|}
\hline Table (1): Baseline Characteristic in the Two Groups \\
\hline & $\begin{array}{c}\text { Agonist group (A) } \\
\mathbf{N}=\mathbf{2 8}\end{array}$ & $\begin{array}{c}\text { Antagonist group (B) } \\
\mathbf{N}=\mathbf{2 9}\end{array}$ & $\mathbf{p}$ \\
\hline $\begin{array}{c}\text { Age(years) Range } \\
\text { Mean } \pm \text { SD }\end{array}$ & $\begin{array}{c}21-35 \\
28.04 \pm 4.43\end{array}$ & $\begin{array}{c}22-34 \\
27.45 \pm 3.66\end{array}$ & 0.587 \\
\hline BMI(kg/m2) & $31.03 \pm 2.34$ & $30.12 \pm 1.25$ & 0.071 \\
\hline $\begin{array}{c}\text { Duration of } \\
\text { infertility(years) }\end{array}$ & $7.2 \pm 3.01$ & $7.31 \pm 3.36$ & 0.893 \\
\hline $\begin{array}{c}\text { Type of infertility } \\
\text { Primary } \\
\text { Secondary }\end{array}$ & $85.7 \%$ & $89.7 \%$ & 0.650 \\
\hline $\begin{array}{c}\text { Basal FSH (IU/ml) } \\
\text { Mean } \pm \text { SD }\end{array}$ & $14.3 \%$ & $10.3 \%$ & 0.112 \\
\hline $\begin{array}{c}\text { Basal LH (IU/ml) } \\
\text { Mean } \pm \text { SD }\end{array}$ & $5.70 \pm 1.53$ & $6.37 \pm 1.57$ & 0.438 \\
\hline $\begin{array}{c}\text { Serum E } \mathbf{2} \text { (pg/ml) } \\
\text { Mean } \pm \text { SD }\end{array}$ & $30.73 \pm 9.8$ & $9.64 \pm 2.16$ & 0.797 \\
\hline
\end{tabular}

There were no statistically significant differences between two groups as regard age, body mass index (BMI), basal FSH, $\mathrm{LH}$, serum $\mathrm{E}_{2}$ at the first day of cycle and type and duration of infertility. Most of the patients were cases of primary infertility in both groups.

Table (2): Duration of Stimulation, Number of $\mathrm{HMG}$ Ampoules, $\mathrm{E}_{2}$ and Endometrial Thickness at hCG Day in the Two Groups.

\begin{tabular}{|c|c|c|c|}
\hline & $\begin{array}{c}\text { Agonist group } \\
\mathbf{N}=\mathbf{2 8}\end{array}$ & $\begin{array}{c}\text { Antagonist group } \\
\mathbf{N}=\mathbf{2 9}\end{array}$ & $\mathbf{p}$ \\
\hline $\begin{array}{c}\text { Duration of stimulation (days) } \\
\text { Mean } \pm \text { SD }\end{array}$ & $11.25 \pm 1.69$ & $9.48 \pm 0.83$ & 0.000 \\
\hline $\begin{array}{c}\text { Number of ampoules of HMG } \\
\begin{array}{c}\text { Serum E2 (pg/ml) } \\
\text { Mean } \pm \text { SD }\end{array}\end{array}$ & $32.46 \pm 7,34$ & $26.59 \pm 2.67$ & 0.000 \\
\hline $\begin{array}{c}\text { Endometrial thickness (mm) } \\
\text { Mean } \pm \text { SD }\end{array}$ & $10.44 \pm .90$ & $2441.55 \pm 493.30$ & 0.003 \\
\hline
\end{tabular}

There were statistically significant differences between both groups as regard the stimulation period, number of ampoules of HMG and Serum $\mathrm{E}_{2}$ level on the day of hCG administration while there were no statistically significant difference between the two groups as regard endometrial thickness at the day of hCG administration. 
Table (3): Number and degree of maturity of retrieved oocytes in the two groups.

\begin{tabular}{|c|c|c|c|}
\hline & $\begin{array}{c}\text { Agonist group (A) } \\
\mathbf{N = 2 8} \\
\text { Mean } \pm \text { SD }\end{array}$ & $\begin{array}{c}\text { Antagonist group (B) } \\
\mathbf{N = 2 9} \\
\text { Mean } \pm \text { SD }\end{array}$ & p \\
\hline $\begin{array}{c}\text { Total number of } \\
\text { oocytes }\end{array}$ & $10.18 \pm 3.9$ & $10.55 \pm 2.92$ & 0.683 \\
\hline $\begin{array}{c}\text { number of GV } \\
\text { oocytes }\end{array}$ & $1.71 \pm 1.61$ & $1.55 \pm 1.24$ & 0.888 \\
\hline $\begin{array}{c}\text { number of MI } \\
\text { oocytes }\end{array}$ & $.75 \pm 1.3$ & $.55 \pm .91$ & 0.985 \\
\hline $\begin{array}{c}\text { number of MII } \\
\text { oocytes }\end{array}$ & $7.71 \pm 2.79$ & $8.85 \pm 2.8$ & 0.326 \\
\hline
\end{tabular}

No statistically significant difference was detected in the total number of oocytes and degree of maturity of retrieved oocytes in the two groups.

Table (4): Fertilization rate in the two groups

\begin{tabular}{|l|c|c|c|}
\hline & $\begin{array}{c}\text { Agonist group } \\
\mathbf{N}=\mathbf{2 8}\end{array}$ & $\begin{array}{c}\text { Antagonist group } \\
\mathbf{N = 2 9}\end{array}$ & $\mathbf{p}$ \\
\hline Fertilization rate & $77.49 \%$ & $87.89 \%$ & 0.004 \\
\hline Implantation rate & $14.99 \%$ & $15.94 \%$ & 0.96 \\
\hline
\end{tabular}

Fertilization rate was higher in group B compared to in group A and the difference between both groups was statistically significant. There was no statistically significant difference implantation rate in the two groups.

Table (5): Number of embryos and their grades in the two groups

\begin{tabular}{|c|c|c|c|}
\hline & $\begin{array}{c}\text { Agonist group } \\
\mathbf{N = 2 8} \\
\text { Mean } \pm \text { SD }\end{array}$ & $\begin{array}{c}\text { Antagonist group } \\
\mathbf{N}=\mathbf{2 9} \\
\text { Mean } \pm \text { SD }\end{array}$ & p \\
\hline Total number of Embryos & $6.12 \pm 2.64$ & $7.38 \pm 2.58$ & 0.072 \\
\hline Grade A Embryos & $4.57 \pm 2.17$ & $6.38 \pm 2.31$ & 0.004 \\
\hline Grade B Embryos & $1.50 \pm 1.75$ & $0.97 \pm 0.73$ & 0.947 \\
\hline
\end{tabular}

There was no statistically significant difference in the total number of embryos in the two groups. The number of grade A embryos was higher in group $\mathrm{B}$ than in group $A$ and this difference was statistically significant. There was no statistically significant difference in the number of Grade B embryos between the two groups.

Table (6): Pregnancy rates in both groups

\begin{tabular}{|c|c|c|c|}
\hline & $\begin{array}{c}\text { Agonist group } \\
\mathbf{N}=\mathbf{2 8}\end{array}$ & $\begin{array}{c}\text { Antagonist group } \\
\mathbf{N = 2 9}\end{array}$ & $\mathbf{p}$ \\
\hline Chemical pregnancy rate & $11 / 28=39.3 \%$ & $10 / 29=34.48 \%$ & 0.71 \\
\hline Clinical pregnancy rate & $9 / 28=32.1 \%$ & $9 / 29=31.00 \%$ & 0.928 \\
\hline
\end{tabular}

There was no statistically significant rates in the two groups difference in Chemical, Clinical pregnancy 
Table (7): Moderate OHSS in both groups

\begin{tabular}{|c|c|c|c|}
\hline & $\begin{array}{c}\text { Agonist group } \\
\mathbf{N}=\mathbf{2 8}\end{array}$ & $\begin{array}{c}\text { Antagonist group } \\
\mathbf{N}=\mathbf{2 9}\end{array}$ & $\mathbf{p}$ \\
\hline Moderate OHSS & $4 / 28=14.29 \%$ & $0 / 29=0 \%$ & 0.035 \\
\hline
\end{tabular}

Moderate OHSS was documented in 4 cycles of group A (14.29\%) but was not documented in any cycle of group B (0 $\%)$. This difference was statistically significant $(p=0.035)$

\section{Discussion:}

Many RCTs have compared the GnRH agonist long luteal protocol to the GnRH antagonist protocol. The antagonist based protocols allowed to reduce the dose of gonadotrophins used and the duration of the stimulation regimens. Besides, the antagonist granted a more physiological pattern of follicular recruitment, with fewer small growing follicles and lower E2 levels, reducing the risk of severe ovarian hyperstimulation syndrome (OHSS). However, the retrieved oocytes were significantly fewer and a trend towards lower pregnancy rate (PR) could be noticed in most of the RCTs ${ }^{\left({ }^{8}\right)}$.

Five of these RCTs were examined in a meta-analysis published in a Cochrane review (Al-Inany $\mathrm{H}$ et al,. 2002) that confirmed the previous findings as regards the duration of the stimulation protocol, the amount of gonadotrophins used, the number of oocytes retrieved and the E2 levels on the day of hCG administration. Additionally, this meta-analysis showed a significant reduction in clinical pregnancy rate and failed to prove a significant preventive effect over severe OHSS ${ }^{(9)}$.

However, a 5\% higher clinical pregnancy rate did not match with an increase in livebirth rate according to a subsequent metaanalysis (Kolibianakis E.M et al,.2006) comparing agonist and antagonist ${ }^{(10)}$.

In addition to that, the greater safety of GnRH antagonist over GnRH agonist has been definitively demonstrated in a recent version of the Cochrane review (Al-Inany $\mathrm{H}$ et al,. 2011) ${ }^{(11)}$, in which a further clinical advantage has been detected, i.e. the reduction in the number of cycles cancelled due to OHSS risk. Furthermore, this review suggested that GnRH antagonist administration provides comparable results to traditional GnRH agonist stimulation, as opposed to previous works. In particular no significant differences concerning live-birth rates, ongoing pregnancy rates, miscarriage rates per clinical pregnancy rate, and rates of cancellation due to poor ovarian response were reported $^{(11)}$.

Despite the undeniable advantages provided by GnRH antagonist, their efficacy is still debated. Therefore, scientific research on the use of GnRH antagonist in $\mathrm{COH}$ for IVF/ICSI has been promoted even in recent years ${ }^{(8)}$.

There were many studies in IVF cycles that compare the long agonist protocol with the antagonist protocol in PCOS patients. Most of these were used antagonist protocols either fixed or flexible and only two were used early initiation of the antagonist protocol $^{(12)}$.

Five of these RCTs were examined in a meta-analysis published by Mancini et al $2010^{(12)}$. The authors in this meta-analysis did not find a statistically significant difference between the use of a GnRH Antagonist protocol versus the standard long protocol in the incidence of pregnancy and of abortion in patients with PCOS undergoing IVF. The Antagonist protocol also seems to reduce significantly the incidence of OHSS. In this metaanalysis, they could not study the total doses of gonadotrophins used, nor the duration of stimulation or the number of cumulus-oocyte complexes. However, previous studies all agreed that the antagonist protocol is more patient friendly. Still, since the antagonist can be started alternatively on the first day of 
stimulation, on day 6 , or according to the follicles diameter, to date no comparative studies have been made in PCOS to determine if there is a protocol that works best for this population ${ }^{(12)}$.

The two Currently comparative studies have been published in PCOS patients between $\mathrm{GnRH}$ agonists and early initiation antagonists (Hwang et al., 2004 (13) and Lainas et al., $\left.2007^{(6)}\right)$. In Hwang et al., $2004^{(13)}$, the GnRH antagonist cetrorelix was started one day prior to initiation of stimulation with HMG and was compared with the long agonist protocol in 60 PCOS patients while in Lainas et al., $2007^{(6)}$, antagonist ganirelix was started in the first day of stimulation and was compared with the long agonist protocol in 78 PCOS who received OC pill treatment for three weeks. In both studies full dose of antagonist ( 1 ampoule) was administered from the start to the end.

The current study is designed to compare long agonist protocol with early initiation of antagonist (day-1 of stimulation) in PCOS patients undergoing ICSI who received OC pretreatement. It is differed in methodology from both studies of early intiation of antagonist protocol in PCOS patients as we start with the half dose (Cetrotide $0.125 \mathrm{ml}$ ) from the first day of stimulation until the leading follicle reached $14 \mathrm{~mm}$ then full dose (Cetrotide $0.25 \mathrm{ml}$ ) upto the day of hCG administration.

The goal of this study is maintaining low LH level throughout the follicular phase. This goal is clearly achieved when using the long GnRH agonist protocol, and it seems that because of these accomplishments the clinical results from the long agonist protocol were superior compared with results from the standard GnRH antagonist protocol.

This modification is similar in concept to the long GnRH agonist protocol and yet maintains the advantages of the GnRH antagonist protocol. The addition of the $\mathrm{GnRH}$ antagonist in early follicular phase of the cycle to the flexible antagonist treatment in this study achieves this goal. We believe that this novel modification in low resources countries optimize follicular recruitment and maximize the results without increase in the cost.

In the current study, the mean duration of stimulation was shorter in group B compared to group A. These results are comparable to results of Hwang et al., 2004 , Lainas et al., 2007 and almost all comparative studies between long agonist ant antagonist protocols. The difference in our study was highly significant and this came in agreement with Lainas et al., 2007. The significant reduction of number of ampoules of gonadotrophins in antagonist group when compared to the agonist group found in our study. Also, these results are comparable to results of Hwang et al., 2004 and most of comparative studies between long agonist and antagonist protocols. The difference in our study was highly significant and this came in agreement Hwang et al., 2004 but Lainas et al., 2007 did not comment on the amount of recombanant FSH (rFSH) used.

Serum $\mathrm{E}_{2}$ at the day of hCG administration was found to be lower in group B compared to group A which support the results of Hwang et al., 2004 and most of comparative studies between long agonist and antagonist protocols. This difference of statistically significant value likes that of Hwang et al., 2004.

In one RCT conducted by Tehraninejad et al,. $2010^{(14)}$, to evaluate the efficacy of antagonist in comparison with the GnRH agonist protocol in OCP pretreated PCOS patients undergoing their first ART cycle. They found that Serum $E_{2}$ at the day of hCG administration was found to be lower in agonist group compared to antagonist group. They explained that, coasting was used in agonist group when serum estradiol was $>3000 \mathrm{pg} / \mathrm{mL}$. This was done in nine cases agonist group but not done in any case of the antagonist group.

$$
\text { Regarding retrieved oocytes }
$$
number, no statistically significant difference was found between the studied 
groups. These findings are in accordance with the results of Hwang et al., 2004, Lainas et al., 2007 and all comparative studies between long agonist and antagonist protocols in PCOS patients included in Mancini et al,. 2010.

In the present study, comparable number of retrieved oocytes and MII oocytes in GnRh antagonist arm can be due to better synchronization of early antral follicles after partial blocking of $\mathrm{GnRH}$ receptor by the half dose of cetritide.

Fertilization rate was lower in group A compared to group B in the current study. This difference was statistically significant in contrast to the results of Hwang et al., 2004, Lainas et al., 2007. This came in agreement with Tehraninejad et al,. 2010, but with no significant difference.

Despite our study did not find statistically significant difference in the number of resulting embryos in the two groups, the number of grade A embryos was higher in group B than in group A and this difference was statistically significant. This is came in agree with Tehraninejad et al,. 2010. They found that the number of good quality embryos transferred was significantly higher in antagonist group. This is in contrast to the RCT conducted by Kurzawa et al,.2008 ${ }^{(15)}$, to evaluate embryological and clinical efficacy of $\mathrm{GnRH}$ antagonist and agonist stimulation protocols in non-obese women with PCOS. They found no difference in good quality embryos between agonist and antagonist group. This is due to their using flexible antagonist protocol for $\mathrm{COH}$ in antagonist arm while we used antagonist from the first day of stimulation which reduce the high LH in the early follicular phase. This may make follicular development more or less optimal.

In the current study, there were no statistically significant differences in the two groups as regard to chemical and clinical pregnancy rates. This is come in agree with the recent version of the Cochrane review (Al-Inany $\mathrm{H}$ et al,. 2011), and most of recent comparable studies between agonist and antagonist. While pregnancy rates in our study were comparable to these of Tehraninejad et al,. 2010 and Hwang et al., 2004, they were lower than that of Lainas et al., 2007 and Kurzawa et al,. 2008. This is may be attributed to the high body mas index (BMI) of our patients since Pregnancy rate was found to be higher in patients with normal BMI than that of high BMI ${ }^{(16)}$.

There was no statistically significant difference in the implantation rate in the two groups in our study. This is comparable to that of Hwang et al., 2004 while it is lower than that of Kurzawa et al,.2008. This is explained by, in Kurzawa et al,.2008, all PCOS women were non obese and absence of use OCP pretreatment.

In the current study, moderate OHSS according to Rizk and Aboulghar (1999) (17) was documented in 4 cycles of group A but was not documentd in any cycle of group $\mathrm{B}$ and this difference was statistically significant. This is come in agree with the last version of the Cochrane review (Al-Inany $\mathrm{H}$ et al,. 2011) and Mancini et al,. 2010.

In summary, this novel antagonist protocol offers a safe and efficient treatment for patients who present with PCOS who are at high risk of OHSS to standard IVF stimulation protocols. Larger RCTs with enough power are needed to further evaluate IVF outcome and the potential benefits of early initiation of GnRHantagonist protocols in PCOs patients.

\section{References:}

\section{Stadtmauer L.A, Sarhan A, Duran} E.H, et al. The impact of a gonadotropin-releasing hormone antagonist on gonadotropin ovulation induction cycles in women with polycystic ovary syndrome: a prospective randomized study. Fertil Steril 2011; 95:216-220.

2. The Thessaloniki ESHRE/ASRMSponsored PCOS Consensus Workshop Group. Consensus on 
infertility treatment related to polycystic ovary syndrome. Fertil Steril 2008; 89:505-522.

3. Esinler I, Bozdag G, Karakocsokmensuer L. Preventing ovarian hyperstimulation syndrome: cabergoline versus coasting. Arch Gynecol Obstet 2013; 288(5):11591163.

4. Copperman A.B \& Benadiva C. Optimal usage of the GnRH antagonists: a review of the literature. Reprod Biolog and Endocrinol 2013; 11:20-27.

5. Ben-Rafael Z. Agonist or antagonist: what is preferable for in vitro fertilization?. Gynecol Endocrinol 2012; 28(1):8-21.

6. Lainas T.G, Petsas G.k, Zorzovilis I.Z, et al. Initiation of GnRH antagonist on Day 1 of stimulation as compared to the long agonist protocol in PCOS patients. A randomized controlled trial: effect on hormonal levels and follicular development. Hum Reprod 2007; 22(6): 1540-1546.

7. Huirne J.A, Homburg R, Lambalk C.B. Are GnRH antagonists comparable to agonists for use in IVF?. Hum Reprod 2007; 22(11):2805-2813.

8. Marci R, Grazlano A, Lo Monte G. $\mathrm{GnRH}$ antagonists in assisted reproductive techniques: a review on the Italian experience. European Review for Medical and Pharmacological Sciences 2013; 17: 853-873.

9.Al-Inany H, Aboulghar M. GnRH antagonist in assisted reproduction: a Cochrane review. Hum Reprod 2002; 17:874-885.

10.Kolibianakis E.M, Collins J, Tarlatzis BC, et al. Among patients treated for IVF with gonadotrophins and GnRH analogues, is the probability of live birth dependent on the type of analogue used? A systematic review and meta-analysis.
Hum Reprod Update 2006; 12:651671.

11. Al-Inany H, Youssef MAFM, Aboulghar M, et al. Gonadotrophinreleasing hormone antagonists for assisted reproductive technology. Cochrane Database Syst Rev 2011; 11 : CD001750.

12. Mancini F, Tur R, Martinez F, et al. Gonadotrophin-releasing hormoneantagonists vs long agonist in in-vitro fertilization patients with polycystic ovary syndrome: a meta-analysis. Gynecol Endocrinol 2010; Early Online:1-6.

13. Hwang J.L, Seow K.M, Lin Y.H, et al. ovarian stimulation by concomitant administration of cetrorelix acetate and HMG following Diane-35 pretreatment for polycystic ovary syndrome: A prospective randomized study. Hum Reprod 2004; 19(9):19932000.

14. Tehraninejad ES, Nasiri R, Rashidi B, et al. Comparison of $\mathrm{GnRH}$ antagonist with long GnRH agonist protocol after OCP pretreatment in PCOs patients. Arch Gynecol Obstet 2010; 282:319-325.

15. Kurzawa R, Ciepiela $P$, Baczkowski $\mathbf{T}$, et al. Comparison of embryological and clinical outcome in GnRH antagonist vs. GnRH agonist protocols for in vitro fertilization in PCOS nonobese patients.

16. Marci R, Lisi F, Soave I, et al. Ovarian stimulation in women with high and normal body mass index: $\mathrm{GnRH}$ agonist versus GnRH antagonist. Gynecol Endocrinol 2012; 28(10):792-795.

17. Rizk B \& Aboulghar $M$. Classification, pathophysiology and management of ovarian hyperstimulation syndrome. In Brinsden P (ed). In vitro fertilization and assisted reproduction. New York, London: The Parthenon Publishing Group 1999:131-155. 